\title{
Normdaten
}

Claus Harringer*

\section{Multiple Persönlichkeiten in Zeiten digitaler Publikationsformen}

\author{
Ein Erfahrungsbericht zur „Open Researcher and Contributer ID“ (ORCID)
}

https://doi.org/10.1515/iwp-2020-2091

\section{Hintergrund ORCID}

Als der - auf talkshowtaugliche Lebensratschläge spezialisierte - Starphilosoph Richard David Precht seinen Bestseller „Wer bin ich - und wenn ja, wie viele?“ verfasste, hatte er mit dem Titel wohl kaum die verwirrende Welt der „Author IDs“ vor Augen. Dennoch formuliert er mit ihm genau die Frage, die sich aufdrängt, wenn man mit verschiedenen Systemen zur Identifizierung von Autor/innen wissenschaftlicher Texte konfrontiert wird.

Wissenschaftliche Autor/innen publizieren in unterschiedlichen Journalen und Verlagen, wechseln ihre Arbeitsplätze, Mitgliedschaften und manchmal auch ihre Namen (etwa heiratsbedingt, was Frauen nach wie vor stärker betrifft). Darum ist es sinnvoll, sie anhand eines (alpha)numerischen Codes identifizieren zu können, der eine Konstante im (heutzutage immer unbeständigeren) akademischen Lebenslauf darstellt und die verschiedenen Affiliationen und Publikationen bündelt. Letztere werden nämlich von unterschiedlichen Literatur- und Zitationsdatenbanken erfasst, was die Sache verkompliziert, da diese oft direkt miteinander konkurrieren.

Die Marktführer sind dabei Scopus (Elsevier) und Web of Science (Clarivate Analytics) und beide haben auch eigene Identifikationssysteme entwickelt. Clarivate Analytics verwendet(e) Researcher ID ${ }^{1}$, seit April 2019 Publon,

1 Nicht zu verwechseln mit dem Projekt „ResearchID“ (https:// researchid.co/), bei dem man (hauptsächlich via „Scopus Author ID“ und Google Scholar Profil) die eigenen Publikationen auf einer Seite versammelt, auf der daraus automatisch Google-Scholar Metriken (h-index, Zitierungen) errechnet werden.

*Kontaktperson: Claus Harringer, B. A., M. A., Johannes Kepler Universität Linz, Institut für Philosophie und Wissenschaftstheorie, Altenberger Straße 69, 4040 Linz, E-Mail: claus.harringer@jku.at und Elsevier die Scopus Author ID. Auch die bibliographische Datenbank MEDLINE bzw. die Suchmaschine PubMed, die über 25 Millionen biomedizinische Journalartikel bzw. die Referenzen auf diese enthält, hat mit Pub Med Author ID Project ein eigenes Identifikationssystem entwickelt. $^{2}$

Dass die Existenz mehrerer Parallelsysteme Probleme verursacht, sollte nicht verwundern: Bei Autor/innen, die in verschiedenen Journalen publizieren, führt das zu entsprechenden Parallelexistenzen. Darum wäre es grundsätzlich eine sinnvolle Sache, wenn es für alle Autor/innen, die wissenschaftlich publizieren, ein einziges System gäbe, anhand dessen sie eindeutig identifizierbar sind. Standardisierung ist zwar kein Allheilmittel (bei wissenschaftlichen Methoden, Theorien, Modellen etc. ist Pluralismus auf jeden Fall vorzuziehen), oft aber die praktikabelste Lösung.

Die EU-Kommission versucht z. B. seit Jahren, Hersteller von Mobiltelefonen zur Vereinheitlichung ihrer Ladegeräte zu bringen, um jährlich 51.000 Tonnen Elektromüll zu vermeiden. Aufgrund dieser Initiative hat sich USB-C als Standard durchgesetzt (nur nicht bei Apple-Geräten, da der Konzern diese Vorgabe als „innovationsfeindlich“ kritisiert). In diesem Fall brauchte es politischen Druck, damit die Beteiligten einen gemeinsamen Standard akzeptieren - das wissenschaftliche Publikationswesen wurde von externen Interventionen aber bislang leider verschont.

Zumindest scheint innerhalb der Branche ein gewisses Problembewusstsein zu bestehen, weshalb 2010 einige Großverlage (Elsevier, Springer, Nature) und Großforschungseinrichtungen (CERN) die Non-Profit-Organisation ORCID gründeten, die seit 2012 die ORCID-IDs (basierend auf der ISO-Norm 27729) vergibt. ${ }^{3}$ Derzeit hat ORCID nach Eigenaussage 1.135 Mitgliedsorganisationen und bis-

2 https://www.nlm.nih.gov/pubs/techbull/nd10/nd10_pm_author_ id.html [18.11.2019].

3 https://de.wikipedia.org/wiki/ORCID 


\begin{tabular}{|c|c|c|c|}
\hline $\begin{array}{l}\text { Claus } \\
\text { Harringer }\end{array}$ & Biography 3 & $\therefore$ & ह 1 \\
\hline \multicolumn{4}{|l|}{ ORCID ID } \\
\hline Chttps://orcid.org/0000-0001-7697-1311 & $\checkmark$ Employment $(0) B$ & + Add employment & lt sort \\
\hline View public version & \multicolumn{3}{|l|}{ Employment lists organizations where you have been professionally affiliated. Add employer. } \\
\hline D Display your ID on other sites 0 & $\checkmark$ Education and qualifications $(0) ?$ & + Add education & It Sort \\
\hline Public record print view 0 & \multicolumn{3}{|l|}{ Education lists the organizations where you were educated. Add education or add qualification. } \\
\hline 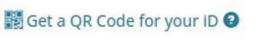 & $\checkmark$ Invited positions and distinctions $(0) ?$ & + Add distinction & It Sort \\
\hline Also known as & \multicolumn{3}{|c|}{$\begin{array}{l}\text { An invited position is an invited non-employment affiliation. A distinction is an honorary or other award, distinction, or prize. Add invited } \\
\text { position or add distinction. }\end{array}$} \\
\hline Country & $\checkmark$ Membership and service $(0) ?$ & + Add membership & It Sort \\
\hline & \multicolumn{3}{|c|}{$\begin{array}{l}\text { Membership is membership in any society or organization. Service is a dedication of time, money, or other resources. Add membership or } \\
\text { add service. }\end{array}$} \\
\hline Keywords & $\sim$ Funding $(0) ?$ & + Add funding & It Sort \\
\hline Websites \& Social Links & \multicolumn{3}{|l|}{ Funding captures grants and other awards you have received to support your research. Add funding. } \\
\hline & \multicolumn{3}{|l|}{ > Works $(0$ of 0$) ?$} \\
\hline \multicolumn{4}{|l|}{ Other IDs } \\
\hline \multicolumn{4}{|l|}{ Scopus Author ID: 57204899626} \\
\hline $\begin{array}{l}\text { Emails } \\
\text { claus.harringer@jku.at }\end{array}$ & & & \\
\hline
\end{tabular}

Abbildung 1: Screenshot der OCRID-Überblicksseite.

lang 8.338.415 ORCID IDs vergeben. ${ }^{4}$ Selbst wenn alle Datenbankbetreiber, Journalverlage und akademische Institutionen weltweit sich beteiligen, besteht das große Problem natürlich darin, die bisher existierenden Identifikationssysteme in das ORCID-System zu überführen. Wenn das nicht gelingt, entsteht lediglich ein neues Element, das die Unübersichtlichkeit noch zusätzlich erhöht.

\section{Praxistest ORCID}

Die Registrierung (als Person oder Institution) auf der Seite (https://orcid.org/signin) ist schnell durchgeführt (30 Sekunden - 1 Minute): Es muss bei einem persönlichen Account lediglich Vorname (Nachname optional) und eine gültige E-Mail-Adresse angegeben werden, sowie ein einigermaßen sicheres Passwort gewählt und durch erneute Angabe bestätigt werden. Nach Auswahl der Sichtbarkeitseinstellungen (Everyone, Trusted parties oder Only me), Akzeptanz von Nutzungsbedingungen sowie Datenschutzrichtlinie und einem CAPTCHA-Häkchen wird eine Bestätigungs-Email verschickt. Nach Anklicken des darin enthalten Links ist der Account erstellt und die Übung im Selbstmanagement kann beginnen:

Die persönlichen Merkmale können über die folgenden Felder eingetragen werden: Employment, Education

4 https://orcid.org/members [9.4.2020]. and qualifications, Invited positions and distinctions, Membership and services, Funding. Jeweils verpflichtend sind Angaben zu den Items Organization, City und Country zu machen, optional kommen Region, Department, Role/Title, URL, sowie Duration hinzu. Das Feld invited distinctions fragt nach Distinction/award) anstelle von Role/Title, das Feld Membership nach Type.

Interessant ist das Feld Funding, da hier die fördernden Organisationen mit Hilfe eines sogenannten ÜberWizard importiert werden müssen. Dieser ÜberWizard basiert auf ÜberResearch, einer globe grants database, die 2015 von einem ehemaligen Elsevier Mitarbeiter namens Christian Herzog gegründet wurde und mit der Datenbanksuchmaschine Dimensions verkoppelt ist. Diese ist wiederum ein Produkt der Technologiefirma Digital Science, welche strategisch in Forschungs-Start-ups investiert und zur Holtzbrinck Publishing Group gehört (MacMillan Publishers, Zeitverlag, Joint Venture mit Springer Nature). Kurz: Angaben zu Förderungen sind nur über einen Account bei einem weiteren System möglich, das letztlich einer Verlagsgruppe gehört, die ihren Umsatz sowohl mit den Büchern von S. Fischer und Rowohlt als auch Onlineratgebern wie gutefrage.net und Netdoktor macht - aber eben auch vermehrt mit wissenschaftlichen Daten.

Das letzte Feld Works bietet die Möglichkeit, die eigenen Publikationen mit der ORCID-ID zu verlinken. Da es sich um das Kernstück von ORCID handelt, das letztlich über die Brauchbarkeit entscheidet, stelle ich das etwas detaillierter dar: 
Die Eintragung der Publikationen wird durch sogenannte Link wizards, internationale Datenbanken für digitale Publikationen, erleichtert, die den Nutzenden in einer Auswahlliste zur Verfügung gestellt werden. Zunächst lassen sich die in Folge angeführten link wizards nach work type (All, Articles, Data, Book, sowie Student Publications) und nach der geographical area (All, Global, Europe, Asia, North America, South America, Australia) filtern. Hier die komplette Liste der wizards mit Kommentar in Klammer:

- Airiti (größte digitale Datenbank Chinas für akademische e-journals)

- BASE-Bielefeld Academic Search Engine (Suchmaschine für 150 Mio. wissenschaftliche Dokumente aus 7.000 Quellen) ${ }^{5}$

- Crossref Metadata Search (Suche nach 50 Mio. Dokumente in den Crossref Metadata records)

- DataCite (2009 von sechs Einrichtungen wie der British Library oder der TIB Hannover gegründete NonProfit Organisation mit dem Ziel, Forschungsdaten besser zugänglich zu machen, indem sie deren Archivierung vernetzt und die Datensätze mit DOIs versieht)

- Deutsche Nationalbibliothek (DNB) (Zentralarchiv aller deutschsprachigen Veröffentlichungen)
- Europe PubMed Central (Open Access Repository für biomedizinische Forschung)

- ISNI (der „International Standard Name Identifier“, der an alle „schöpferisch aktiven“ natürlichen Personen - nicht nur Autorinnen und Autoren - vergeben werden kann. $)^{6}$

- KoreaMed (Koreanische Assoziation von MedizinJournalen)

- MLA International Bibliography (Fachbibliothek für moderne Philologien)

- Research Data Australia (Forschungsdatensammlung von über 100 australischen Forschungsorganisationen, Regierungsbehörden und Kultureinrichtungen) ${ }^{7}$

- ResearcherID (System zur Autorinnen- und AutorenIdentifikation, 2008 von Thomson Reuters entwickelt und ins „Web of Science“ implementiert)

- Scopus - Elsevier (Profile von Elsevier in der Datenbank Scopus, s.u.)

Da ich über lediglich eine indexierte Publikation verfüge, versuche ich die Integration über Scopus von Elsevier. Das funktioniert auf Anhieb tadellos ${ }^{8}$. Hier die Bildstrecke: 


\section{Scopus ORCID}

1 Select profiles

\section{Select your Scopus profiles}

Please select all profiles that contain publications authored by you and click the next button to continue

\begin{tabular}{|c|c|c|c|c|c|c|c|c|}
\hline \multicolumn{9}{|c|}{ You searched for: Authorname (Harringer, Claus) I edit } \\
\hline & & & & Sort by & \multicolumn{4}{|l|}{ Relevancy } \\
\hline All $\square$ & Authors & Documents & Subject area & \multicolumn{3}{|l|}{ Affiliation } & City & Country \\
\hline \multirow[t]{3}{*}{1} & Harringer, Claus & 百 1 & Computer Science, Social Sciences & Johannes $\mathrm{Ke}$ & ler Universitat Linz & & Linz & Austria \\
\hline & \multicolumn{8}{|c|}{ [F Show recent documents } \\
\hline & & & & & & back & I & Next \\
\hline
\end{tabular}

\section{Scopus ORCID}

1 Select profiles

\section{Select the preferred profile name}

Please select the preferred name for your unique author profile.

Profile name Harringer, Claus $\vee$

\section{Scopus ORCID}

$1 \mid$ Select profiles $>2 \mid$ Select profile name $>3 \mid$ Review publications $>4 \mid$ Review profile $>5 \mid$ Send Author ID $>6$ ISend publications

\section{Review your authored publications}

Please indicate below if this publication is authored by you.

\begin{tabular}{|c|c|c|c|c|c|c|c|}
\hline & & & & & Sort by & Date (Newest) & $\checkmark$ \\
\hline (3) & Document Title & Author(s) & Date & & \multicolumn{3}{|c|}{ Source Title } \\
\hline (2) & \multicolumn{3}{|c|}{$\begin{array}{l}\text { "Good Bot, Bad Bot"? : On problematic areas of Bot-Ontologies,"Good Bot, Bad Bot"?: Zur } \\
\text { Problematik von Bot-Ontologien } \\
\text { View in Scopus }\end{array}$} & $\begin{array}{l}\text { Harringer, } \\
\text { C. }\end{array}$ & 2018 & \multicolumn{2}{|c|}{$\begin{array}{l}\text { Information-Wissenschaft und Praxis } \\
69(5-6), p p .257\end{array}$} \\
\hline \multicolumn{8}{|c|}{ Q. Search for missing documents I back | } \\
\hline
\end{tabular}




\section{Scopus $\mid$ ORCID}

3 I Review publications

4 | Review profile

5 I Send Author ID

6 ISend publications

\section{Review the Scopus profile}

Please review the information below to ensure that the data to be sent to ORCID is correct.

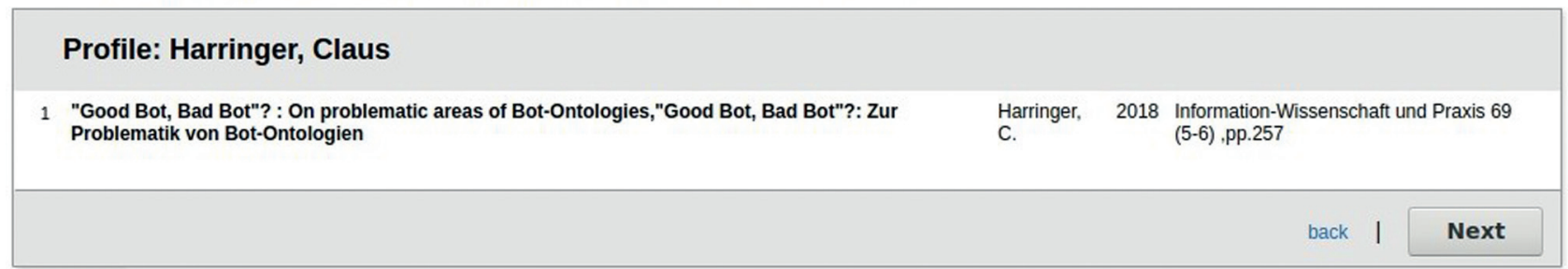

\section{Scopus ORCID}

$1|\underline{\text { Select profiles }}>2| \underline{\text { Select profile name }}>3|\underline{\text { Review publications }}>4| \underline{\text { Review profile }}>5 \mid$ Send Author ID $>$ 6ISend publications

\section{Send the Scopus ID to ORCID}

Thank you for checking your profile. Your Scopus ID is now ready to be sent to ORCID.

\begin{tabular}{|l|l|l|}
\hline E-mail & \multicolumn{2}{|l|}{ Claus.harringer@jku.at } \\
Please enter your institutional or professional email address (e.g. name@university.edu). \\
Confirm E-mail" & Claus.harringer@jku.at \\
\hline & & back I Send Author ID \\
\hline
\end{tabular}

\section{Scopus $\mid$ ORCID}

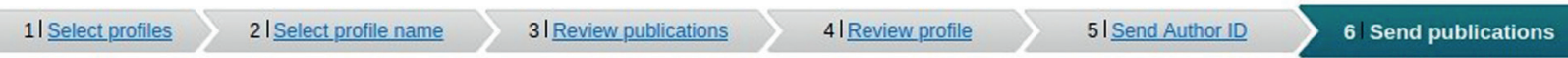

\section{Scopus Author ID submitted}

Your Author ID has been sent to ORCID. However, before we can send your publication list we must ask your permission.

If you wish to send your list of publications to ORCID, please continue to the next step. Alternatively you can return to ORCID.

Abbildung 2: Screenshot der Integration von Publikationen über Scopus von Elsevier. 
Nach Rückkehr zur Überblicksseite sieht diese dann so aus:

$\checkmark$ Employment (0) $?$

+ Add employment It Sort

Employment lists organizations where you have been professionally affiliated. Add employer.

$\checkmark$ Education and qualifications (0) ?

+ Add qualification + Add education It Sort

Education lists the organizations where you were educated. Add education or add qualification.

$\checkmark$ Invited positions and distinctions $(0) ?$

+ Add invited position $\quad$ + Add distinction $1 \uparrow$ sort

An invited position is an invited non-employment affiliation. A distinction is an honorary or other award, distinction, or prize. Add invited position or add distinction.

$\checkmark$ Membership and service (0) 3

+ Add service + Add membership It Sort

Membership is membership in any society or organization. Service is a dedication of time, money, or other resources. Add membership or add service.

$\checkmark$ Funding (0) ?

+ Add funding It Sort

Funding captures grants and other awards you have received to support your research. Add funding.

\begin{tabular}{l|l|l|l|}
$\checkmark$ Works $(\mathbf{1}$ of 1$)$ ? & + Add works & \pm Export works & It Sort \\
\hline
\end{tabular}

y combine 而Delete in $\delta$

"Good Bot, Bad Bot"? : On problematic areas of Bot-Ontologies, "Good Bot, Bad

Bot"?: Zur Problematik von Bot-Ontologien

Information-Wissenschaft und Praxis

2018 | journal-article

DOI: $10.1515 /$ iwp-2018-0040

EID: 2-s2.0-85057761440

Source: Claus Harringer via Scopus - Elsevier

$\star$ Preferred source

$g^{+}$荓

Abbildung 3: Screenshot der OCRID-Überblicksseite nach Integration der Publikation.

In dem Fall ging es relativ einfach - interessanterweise hat das System den englischen Titel des deutschsprachigen Textes hier erstgereiht. Was aber wäre, wenn zusätzlich zu den Scopus-Daten noch die des Konkurrenten Web of Science integriert werden müssten? Darüber kann ich nur Vermutungen anstellen, ebenso wie über die Integrationsfunktion der anderen Datensätze. Im Falle von Web of Science wurde es zunächst so gehandhabt, dass die Autorinnen und Autoren selbst ihre ORCID-ID mit der ResearchID verlinken können - mittlerweile werden Web of Science monatlich aktualisierte ORCID-Daten übermittelt, die dann eingepflegt werden. ${ }^{9}$ Das klingt zunächst sinnvoll - eine genauere Betrachtung legt aber Bedenken nahe: Web of Science hat die ResearchID April dieses Jahres in ein neues System namens „Publon“

9 https://support.clarivate.com/ScientificandAcademicResearch/s/ article/Web-of-Science-Inclusion-of-ORCID-numbers?language=en US [18.11.2019]. überführt, ${ }^{10}$ die Frage ist also, ob die Daten korrekt migriert wurden.

Das ist ein generelles Problem: ORCID ist darauf angewiesen, dass die Publikationsdaten korrekt sind. Wenn das nicht der Fall ist, stellt sich die Frage, wie zielführend Korrekturen im ORCID Tool selbst sind, oder ob man die Metadaten in den integrierten/verlinkten Datenbanken korrigieren müsste, da die Fehler ansonsten bei jeder Aktualisierung erneut auftreten. Auch hier kann ich nur von Scopus sprechen: Der Vorteil dieser Datenbank ist, dass jeder Autor und jede Autorin, mit zumindest einer indexierten Publikation auch ohne Zugangslizenz ein Profil bei Scopus erstellen kann. Darüber ist es z. B. möglich, Zusammenführungen (merging) von Profilen zu beantragen. ${ }^{11}$ Die Suche nach Autor/innen ist generell frei zu-

$10 \mathrm{https}$ //publons.freshdesk.com/support/solutions/arti cles/12000055561-what-is-happening-to-researcherid- [18.11.2019]. 11 https://service.elsevier.com/app/answers/list/c/10546/suppor thub/scopus/ [18.11.2019]. 
gänglich, wodurch sich Phantome wie Mehrfacheinträge recht leicht aufspüren lassen, v.a. jene, die durch unterschiedliche Affiliationen entstehen.

Wenn man in der frei zugänglichen Scopus-Suchfunktion nach Autorinnen- oder Autoren-Profilen ${ }^{12}$ sucht, z.B. nach Pierre Bourdieu (Author last name: Bourdieu, Author first name: P.), bekommt man folgende Treffer:

Tabelle: Treffer der Scopus-Suche nach Pierre Bourdieu. (C. Harringer)

\begin{tabular}{|c|c|c|c|c|c|}
\hline Author & Documents & h-index & Affiliation & City & $\begin{array}{l}\text { Country/ } \\
\text { Territory }\end{array}$ \\
\hline $\begin{array}{l}\text { 1. Bourdieu, } \\
\text { Pierre }\end{array}$ & 84 & 34 & $\begin{array}{l}\text { École Pratique } \\
\text { des Hautes } \\
\text { Etudes Paris } \\
\text { France }\end{array}$ & Paris & France \\
\hline $\begin{array}{l}\text { 2. Bourdieu, } \\
\text { Pierre }\end{array}$ & 6 & 5 & $\begin{array}{l}\text { College de } \\
\text { France }\end{array}$ & Paris & France \\
\hline $\begin{array}{l}\text { 3. Bourdieu, } \\
\text { Pierre }\end{array}$ & 4 & 4 & $\begin{array}{l}\text { EPHE École } \\
\text { Pratique des } \\
\text { Hautes Etudes }\end{array}$ & Paris & France \\
\hline $\begin{array}{l}\text { 4. Bourdieu, } \\
\text { J.P. }\end{array}$ & 1 & 1 & & & \\
\hline $\begin{array}{l}\text { 5. Bourdieu, } \\
\text { P. }\end{array}$ & 1 & 0 & & & \\
\hline $\begin{array}{l}\text { 6. Bourdieu, } \\
\text { Pierre }\end{array}$ & 1 & 1 & & & \\
\hline $\begin{array}{l}\text { 7. Bourdieu, } \\
\text { Plerre }\end{array}$ & 1 & 1 & & & \\
\hline
\end{tabular}

Bei den ersten drei Einträgen kann man sicher sein, dass es sich um dieselbe Person handelt, die nur aufgrund wechselnder Affiliationen mehrfach „existiert“. Auch Treffer Nr. 5 \& 6 müssten eigentlich dazugezählt werden und Treffer 7 dürfte dem geschuldet sein, was Terje Tüür-Fröhlich als „trivial error"13 bezeichnet: Einem einzelnen Tippfehler, der dann ein Phantom erzeugt.

Ein Screenshot der ersten drei Einträge (s. Abb. 4) zeigt:

Autorinnen und Autoren müssen sich auf jeden Fall zuerst darum kümmern, dass die eigenen Publikationsdaten in den Ursprungsdatenbanken korrekt sind, bevor sie mittels der ORCID-Wizards integriert werden. Damit ist abschließend angesprochen, dass Glanz und Elend von

12 https://www.scopus.com/freelookup/form/author.uri [20.11.2019]. 13 Tüür-Fröhlich, Terje (2016): The Non-trivial Effects of Trivial Erro rs in Scientific Communication and Evaluation. Preface: Volker Gade nne. Schriften zur Informationswissenschaft; Bd. 69. Glückstadt/D: vwh. ISBN-10: 3864881048; ISBN-13: 978-3864881046.
ORCID nahe beisammen liegen: Sind die Daten korrekt, ist die Zusammenführung praktisch - man muss sich aber selbst darum kümmern. Im Falle Bourdieus müsste also jemand für den mittlerweile verstorbenen Soziologen und Philosophen ein Autorenprofil anlegen und sich um die Zusammenführung der Einträge bemühen, da ansonsten Zitierungen gesplittet werden (und damit verloren gehen) und der h-Index sinkt.

Letztlich bedeutet dies - frei nach U. Bröckling - einen weiteren Schritt zum unternehmerischen akademischen Selbst. ${ }^{14}$

\section{Nachsatz}

Es war problemlos möglich, eine ORCID-ID für Pierre Bourdieu auszustellen und zumindest alle Scopus-indexierten Publikationen dafür zu reklamieren (https://or cid.org/0000-0001-6386-4269):

Hieran anknüpfend könnte man jetzt Pierre Bourdieu ins Editorial Board der IWP berufen oder zum Angestellten der Johannes Kepler Universität machen.

Dagegen hätte diese vermutlich wenig einzuwenden andere Universitäten gehen zur Verbesserung ihre Metriken sogar den Weg der Scheinanstellung internationaler Topforschender mit beeindruckendem h-Index. Künftig genügt es vielleicht, sie virtuell einzukaufen. Die einzige Möglichkeit, derartigen Missbrauch zu minimieren, besteht in irgendeiner Form der Identitätsfeststellung. Damit verringert sich zwar die Zugänglichkeit des Systems enorm, was seinen Reiz deutlich mindert - geschieht dies aber nicht, ist es nur eine Frage der Zeit, bis sich ungewollte Effekte zeigen.

Deskriptoren: Autor, Identifikation, Name, Name Authority File, ORCID

\section{Literatur}

Bröckling, Ulrich (2007): Das unternehmerische Selbst. Soziologie einer Subjektivierungsform. Frankfurt a. M.: Suhrkamp

Tüür-Fröhlich, Terje (2016): The Non-trivial Effects of Trivial Errors in Scientific Communication and Evaluation. Preface: Volker Gade nne. Schriften zur Informationswissenschaft; Bd. 69. Glückstadt/D: vwh. ISBN-10: 3864881048; ISBN-13: 978-3864881046

14 Bröckling, Ulrich (2007): Das unternehmerische Selbst. Soziologie einer Subjektivierungsform. Frankfurt a. M.: Suhrkamp. 


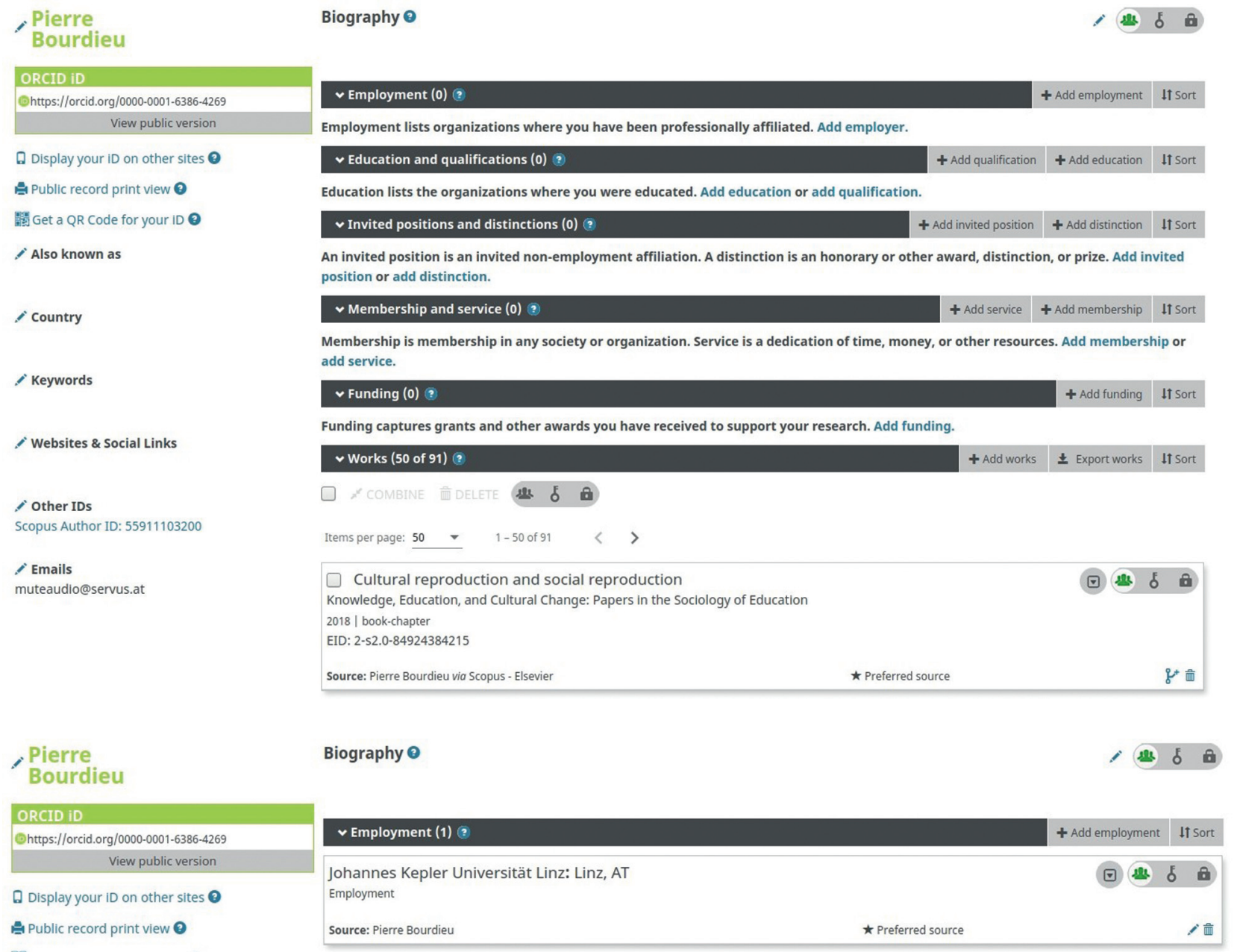

Abbildung 4: Pierre Bourdieu als Mitarbeiter der Johannes Kepler Universität Linz? ORCID iD macht es möglich!

\section{Claus Harringer, B. A., M. A.}

Johannes Kepler Universität Linz

Institut für Philosophie und Wissenschaftstheorie

Altenberger Straße 69

4040 Linz

claus.harringer@jku.at

https://orcid.org/0000-0001-7697-1311

Claus Harringer ist Lektor am Institut für Philosophie und Wissenschaftstheorie an der Johannes Kepler Universität Linz. 